\title{
Evaluation of colloidal silica obtained via the co-precipitation method using octane as an organic phase
}

\author{
Teofil Jesionowski, Beata Tepper, Andrzej Krysztafkiewicz \\ Poznań University of Technology, Institute of Chemical Technology and Engineering, pl. M. Skłodowskiej-Curie 2, \\ 60-965 Poznań, Poland, e-mail: teofil.jesionowski@put.poznan.pl
}

\begin{abstract}
The studies were conducted on the production of silica from emulsion systems by co-precipitation from solutions of sodium metasilicate and hydrochloric acid and octane were used as an organic medium. The obtained products were subjected to a comprehensive physicochemical analysis in order to define their dispersive and morphological properties. Particle diameters and polydispersity were determined by the NIBS (non-invasive back scattering) method while the shape and morphology of the particles were established using scanning electron microscopy (SEM). In order to determine the adsorptive properties of the obtained monodisperse silicas, isotherms of nitrogen adsorption/desorption on their surfaces were determined. Using the isotherms, also the specific surface area, the diameter and the volume of the pores were established. In order to define the hydrophilic/hydrophobic character of the products, profiles of wettability and of sedimentation in water were examined. The obtained products were well wettable with water and an increase in their weight depended on the amount of the applied surfactants. The particles of the precipitated silicas manifested a defined, spherical shape and showed a relatively low polydispersity.
\end{abstract}

Keywords: precipitated silica, emulsion, PSD, SEM, sedimentation, wettability.

Presented at VII Conference Wasteless Technologies and Waste Management in Chemical Industry and Agriculture, Międzyzdroje, 12 - 15 June, 2007.

\section{INTRODUCTION}

Traditional techniques of colloidal silica production are based on reactions of flame hydrolysis ${ }^{1}$, on precipitation from aqueous solutions of alkali metal silicates ${ }^{2-5}$ or on the hydrolysis and condensation of silicic acid esters (Stöber's method). ${ }^{6,7}$

In the age of nanotechnology development and material engineering new, alternate solutions are looked for in the technology of formation of silicas of the defined surface and dispersive properties.

Recognition of such parameters as the size and shape of the particles, their hydrophilic/hydrophobic properties and the specific surface area determines the potential of using the precipitated silicas, particularly as adsorbents and strengthening fillers of polymers.

In silica production by the precipitation technique, the dominating effect on the properties of the product is exerted by a selection of technological parameters. Therefore, in this study an attempt was made to precipitate from the emulsion medium the silicas of the spherical particle shape and restricted surface hydrophilicity.

\section{EXPERIMENTAL}

Silicas were precipitated from aqueous solutions of sodium metasilicatev (VITROSILICON S.A.) and of hydrochloric acid (POCh S.A.). The process was conducted in an emulsion medium. The medium consisted of two emulsions, of which one (E1) contained in its constant composition the sodium metasilicate solution and octane (POCh S.A.). The emulsion was additionally supplemented with a non-ionic surfactant, Rokanol K7 (PCC ROKITA S.A.). The other emulsion (E2) in its constant composition contained the solution of hydrochloric acid and the same organic solvent. In this case the lower amounts of the emulsifier were added. The prepared in this way emulsions were ready for precipitation. Emulsion E2 was placed in a container and subjected to intense mixing using a homogenizer (19 $000 \mathrm{rpm})$. Emulsion E1 was dosed to emulsion $\mathrm{E} 2$ at a constant rate $\left(25 \mathrm{~cm}^{3} / \mathrm{min}\right)$. The reaction taking place in the reactor resulted in an emulsion with the silica contained in it. The emulsion was destabilized by heating. Then, octane was separated by distillation. The obtained product was filtered and the product was dried at the temperature of $105^{\circ} \mathrm{C}$.

The first step of physicochemical analysis of the precipitated silicas involved a determination of the particle size, the parameter of principal importance for the definition of the activity of the obtained powders. Particle size distribution was established using the Zetasizer Nano ZS (Malvern Instruments Ltd.) using the non-invasive back scattering method (NIBS). The particle size distribution permitted us to determine polydispersity as an exponent of the uniformity of filler particles in the studied $\mathrm{SiO}_{2}$. The next stage involved studies on the morphology and microstructure aimed at obtaining the data on the dispersion and structure of individual particles of the precipitated silicas. The studies took advantage of a scanning electron microscope (Philips SEM 515). Moreover, the sedimentation rate and wettability with water were determined using the K100 tensiometer (Krüss). In order to define the adsorptive properties of the studied silicas, isotherms of nitrogen adsorption/desorption were determined. The measurements were performed using the ASAP 2010 apparatus (Micromeritics Instruments Co).

\section{RESULTS AND DISCUSSION}

In the process of the synthesis of highly dispersed silicas selection of technological parameters and amounts of substrates used for the preparation of emulsions in par- 
Table 1. The mean values of particle diameters in the polydispersity of silicas as related to the content of the two emulsifiers in both emulsions

\begin{tabular}{|l|c|c|c|c|c|}
\hline \multirow{2}{*}{ Sample No. } & \multicolumn{2}{|c|}{ Emulsifier content, $\mathrm{g}$} & \multicolumn{2}{|c|}{ Range of particle diameters, $\mathrm{nm}$} & \multirow{2}{*}{ Polydispersity } \\
\cline { 2 - 6 } & $E 1$ & $E 2$ & by intensity & $1280-3090$ & 0.509 \\
\hline 1 & 2.0 & 0.7 & $1480-2670$ & $79-142 ;$ & 1.000 \\
\hline 2 & 1.5 & 0.6 & $91 ; 122-142 ; 220,3680-5560$ & $3090-6440$ & 0.469 \\
\hline 3 & 3.0 & 0.7 & $220-255 ; 396-459 ; 2670-3660$ & $190-295 ; 396-531 ; 2300-4150$ & 290 \\
\hline 4 & 3.0 & 0.8 & $342-615$ & $295-712$ & 0.259 \\
\hline
\end{tabular}

a)

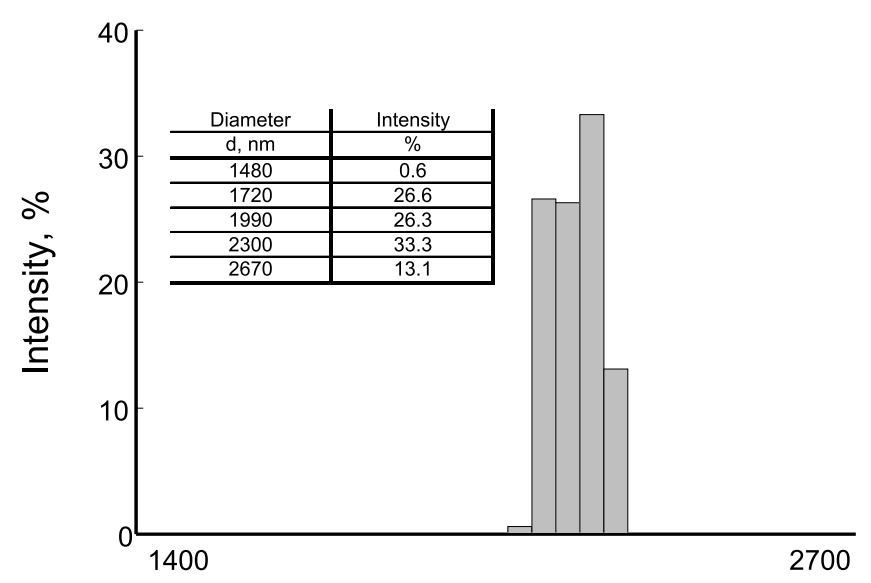

Diameter, $\mathrm{nm}$ b)

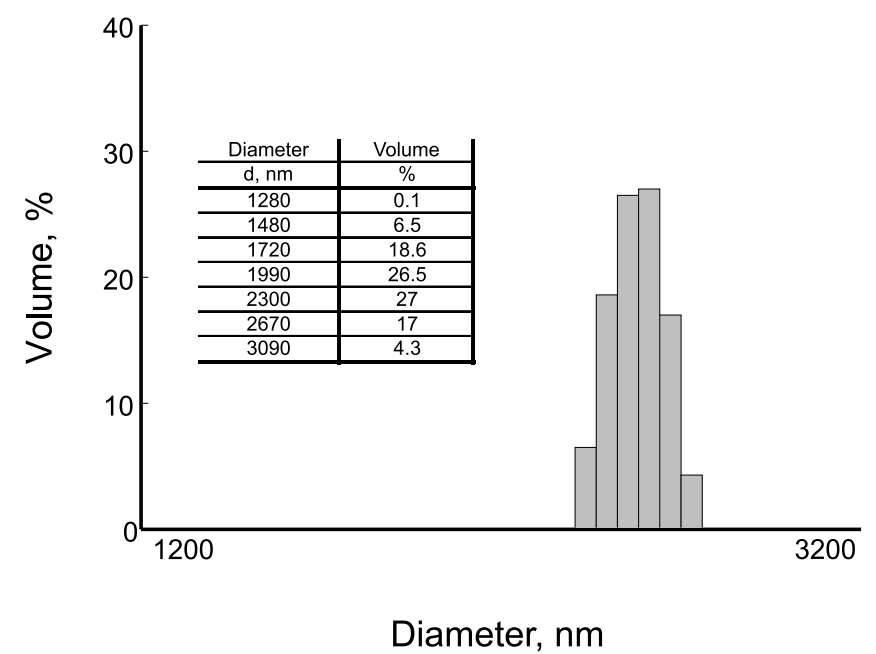

Figure 1. Particle size distribution of the silica sample 1: (a) by intensity and (b) by volume

a)

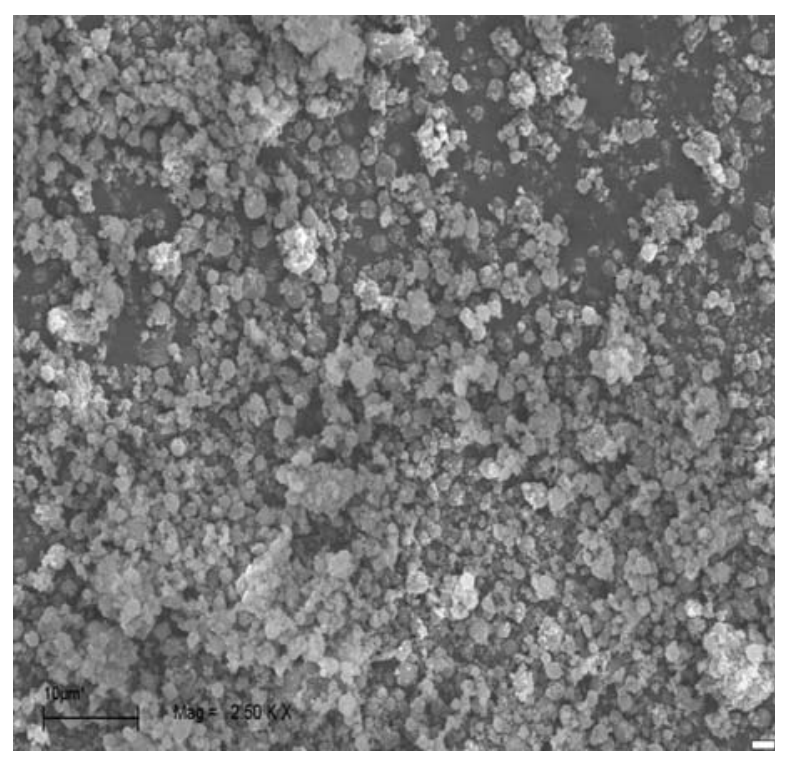

b)

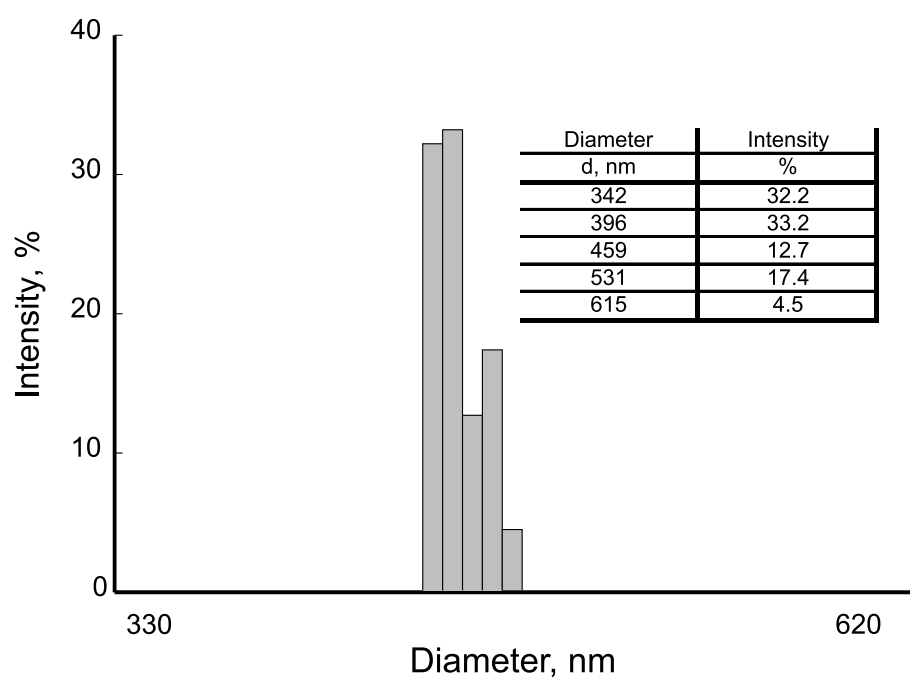

(a) the SEM image and (b) PSD by intensity

Particle size distribution for the silica precipitated in sample 1 is presented in Fig.1.

In the particle size distribution which takes into account band intensity (Fig.1a) a single band was noted. It fitted the range of high diameters $(1480-2670 \mathrm{~nm})$, with the maximum intensity of 26.6 corresponding to the particles of 1720 $\mathrm{nm}$ in diameter. The relatively low value of polydispersity (0.509) pointed to the presence of a single particle group only. In the analysis of the particle size distribution, examined by volume, also a single band was noted but exhibiting a slightly broader range $(1280-3090 \mathrm{~nm})$. 
The least favourable dispersive properties were shown by the silica precipitated in sample 2 . The particle size distribution allowed us to determine the polydispersity which manifested the highest value of 1.000 . The obtained results justified the conclusion that insufficient amount an emulsifier used for the preparation of both emulsions exerted a negative effect on the properties of the product. In the precipitated silica the particles which exhibited a wide range of diameters, dominated. In the particle size distribution taking into account the band intensity, the presence of four bands could be noted (Table 1).

In turn, the SEM image and particle size distribution, when taking into account the band intensity for the precipitated silica sample 4, are shown in Fig.2.

The uniform character of the sample was confirmed by the presence of primary agglomerates both in the size distribution based on the intensity of the bands and in that taking into account the volume share of the bands (Table 1). Small particles dominated, of the diameters fitting the ranges of, $342-615 \mathrm{~nm}$ (by intensity) and $295-712 \mathrm{~nm}$ (by volume), respectively. The sample manifested a relatively low value of polydispersity (0.259).

The effect of the amount of the applied emulsifier on the sedimentation rate of the precipitated silicon dioxide in water is illustrated in Fig.3.

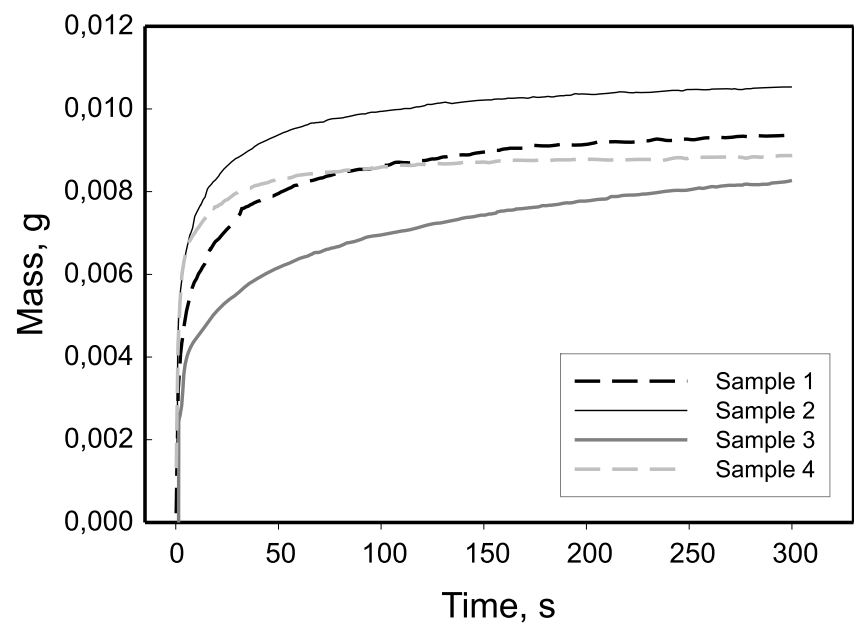

Figure 3. Time dependence of the sedimentation profile in water for the silicas obtained from various organic phases

The highest weight increased vs. time was manifested by the silica precipitated in sample 2. In preparation of both emulsions the lowest amounts of emulsifier were used ( $1.5 \mathrm{~g}$ and $0.6 \mathrm{~g}$, respectively). The weight stabilization of the obtained sediment was observed at the end of measurements, following $250 \mathrm{~s}$. On the other hand, the lowest weight gain was shown by the silica precipitated in sample 3 , in which doubling the amount of the emulsifier in the alkaline emulsion (E1) resulted in the appearance in the particle size distribution of three bands (Table 1).

Wettability of water of the precipitated products is compared in Fig.4.

The silica precipitated in sample 4 (with the highest amount of the applied emulsifier) showed the highest wettability with water which indicated that its surface character was most similar to the hydrophilic one. The course of the curve clearly differed from that of the remaining samples. On the other hand, silicon dioxide pre-

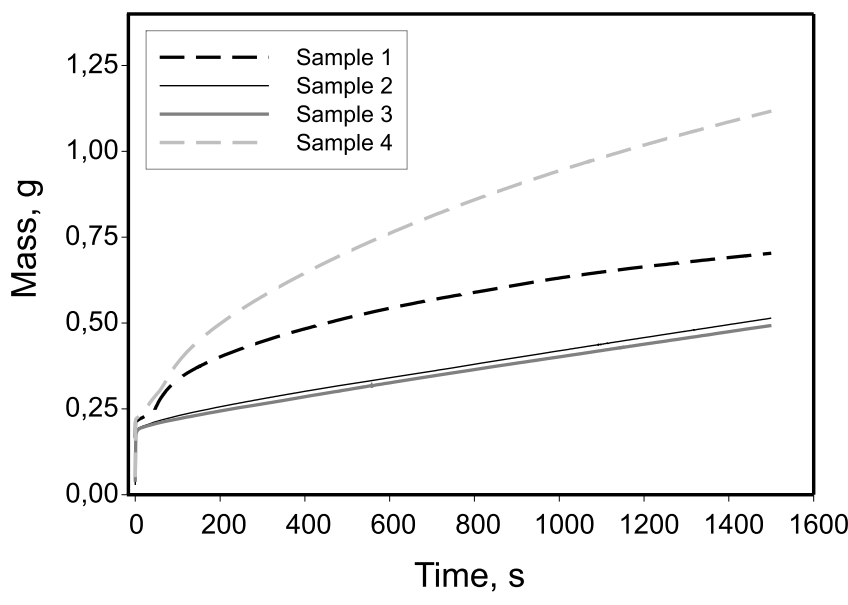

Figure 4. Dependence of wettability vs. time with water of the silicas produced using various organic phases

pared in sample 1 manifested the character resembling that of the preceding silica, although its hydrophilicity was much lower. The remaining silicas showed the minimum tendency to adsorb water which again stressed the hydrophobic character of the surface in the precipitated products.

Nitrogen adsorption/desorption isotherms for the precipitated silicas are shown in Fig.5. The values of the variable such as the specific surface area, the pore volume and the mean pore diameter, are also presented.

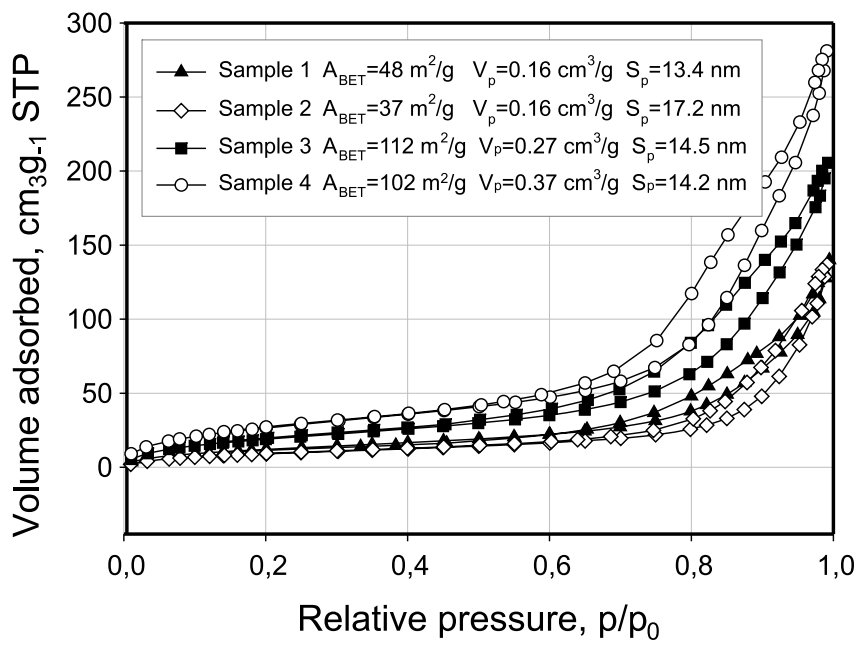

Figure 5. Nitrogen adsorption/desorption isotherms on the surface of the silicas precipitated from an emulsion medium

The profile of the isotherms for silicas precipitated in samples 3 and 4 pointed to their mesoporous character. The amount of adsorbed nitrogen did not increase until the relative pressure exceeded 0.8 and amounted to, 200 $\mathrm{cm}^{3} / \mathrm{g}$ for sample 3 and almost $300 \mathrm{~cm}^{3} / \mathrm{g}$ for sample 4 , respectively. The obtained results indicated a significant effect of the augmented amounts of the applied surfactants on the course of nitrogen adsorption/desorption. As compared to the silicas precipitated in samples 1 and 2, an evident increase in the BET surface, in the range of histeresis loop and in pore volume was observed (Fig.5).

\section{CONCLUSIONS}

The performed studies on the precipitation of monodisperse silica according to the modified technique 
with two emulsions and octane as an organic phase and in the presence of a non-ionic surfactant, Rokanol K7, permitted to obtain silica particles of a relatively high extent of dispersion, spherical shape and low diameters. The results of the studies on the sedimentation and the silica particle wettabilility showed that the lowest weight gain was demonstrated by $\mathrm{SiO}_{2}$ precipitated using the lowest amount of emulsifiers.

The physicochemical properties of the precipitated silicas depend upon the type and amount of the applied precipitating agents and, first of all, on the weight share of the emulsifiers in the two emulsions.

The studies on the adsorptive properties proved that the silicas precipitated from the emulsion medium using the $5 \%$ hydrochloric acid, represented a class of typical mesoporous adsorbents.

\section{ACKNOWLEDGEMENTS}

This work was supported by the Ministry for Science and Higher Education research grant No. 1 T09B 03130 (2006 - 2008).

\section{LITERATURE CITED}

(1) Gun'ko V. M., Mironyuk I. F., Zarko V. I., Turov V. V., Voronin E. F., Pakhlov E. M., Goncharuk E. V., Leboda R., Skubiszewska-Zięba J., Janusz W., Chibowski S., Levchuk Yu. N., Kluyeva A. V.: Fumed silicas prossessing different morphology and hydrophilicity, J. Colloid Interface Sci., 2001, 242, 90 .

(2) Sierra L., Guth J-L.: Synthesis of mesoporous silica with tunable pore size from sodium silicate solutions and a polyethylene oxide surfactants, Microporous Mesoporous Mater., 1999, 27, 243.

(3) Schlomach J, Kind M.: Investigation on the semibatch precipitation of silica, J. Colloid Interface Sci., 2004, 277, 316.

(4) Żurawska J., Krysztafkiewicz A., Jesionowski T.: Active silicas obtained in processes of precipitation from solutions of sodium metasilicate and ammonium chloride, J. Chem. Technol. Biot., 2003, 78, 534.

(5) Jesionowski T., Pokora M., Tylus W., Dec A., Krysztafkiewicz A.: Effect of N-2-(aminoethyl)-3aminopropyltrimethoxysilane surface modification and C.I. Acid Red 18 dye adsorption on physicochemical properties of silicas precipitated in an emulsion route, used as a pigment and filler in acrylic pants, Dyes Pigments 2003, 57, 29.

(6) Stöber W., Fink A., Bohn E.: Controlled growth of monodisperse silica spheres in the micron size range, J. Colloid Interface Sci., 1968, 26, 62.

(7) van Blaaderen A., van Geest J., Vrij A.: Monodisperse colloidal silica spheres from tetraalkoxysilanes: particle formation and growth mechanism, J. Colloid Interface Sci., 1992, 154, 481. 